\title{
ICAM-1 regulates the survival of influenza virus in lung epithelial cells during the early stages of infection
}

\author{
Sreekumar Othumpangat ${ }^{\star}$, John D. Noti, Cynthia M. McMillen, and Donald H. Beezhold \\ Allergy and Clinical Immunology Branch, Health Effects Laboratory Division, National Institute for \\ Occupational Safety and Health, Centers for Disease Control and Prevention, Morgantown, WV, \\ USA
}

\begin{abstract}
Intercellular cell adhesion molecule-1 (ICAM-1) is an inducible cell surface glycoprotein that is expressed on many cell types. Influenza virus infection enhanced ICAM-1 expression and messenger RNA levels. Human bronchial epithelial cells (HBEpC) and nasal epithelial cells, on exposure to different strains of influenza virus (H1N1, H3N2, and H9N1) showed significant increase in ICAM-1 gene expression $(p<0.001)$ along with the ICAM-1 protein levels (surface and secreted). Depleting ICAM-1 in HBEpC with ICAM-1 siRNA and subsequently infecting with H1N1 showed increased viral copy numbers. Influenza virus infection in HBEpC resulted in upregulation of NF- $\kappa B$ protein and the lack of ICAM-1 decreased NF- $\kappa B$ activity in NF- $\kappa B$ luciferase reporter assay. Addition of exogenous IL- $1 \beta$ to HBEpC induced the ICAM-1 expression and decreased matrix gene copy number. Taken together, HBEpC induced ICAM-1 plays a key role in modulating the influenza virus survival possibly through the NF- $\kappa \mathrm{B}$ pathway.
\end{abstract}

\section{Keywords}

Intercellular cell adhesion molecule; Influenza virus; Lung epithelial cells; Signaling; NF- $\kappa B$

\section{Introduction}

Influenza A virus is a negative-strand RNA virus that is among the most common pathogens and poses a continuous threat to human health (Stohr, 2002). Influenza A viruses causing acute infections, continuously escape from recognition by virus neutralizing antibody as a result of antigenic drift or shift (Doherty et al., 2006). Even though predicting the severity of continuously emerging human influenza virus strains remains a high priority, it is limited by our incomplete understanding of the molecular determinants of pathogenicity in influenza infections. Although there are several factors that determine the severity of viral infection, interaction between inherent viral properties and host cellular response ultimately determines disease outcome. A major site of viral contact with the host, and main target of infection and inflammation, is the airway epithelium (Kagnoff and Eckmann, 1997). Epithelial cells in the airways have a variety of inflammatory and immune defense

*Correspondence to: Allergy and Clinical Immunology Branch, National Institute for Occupational Safety and Health, 1095 Willowdale Road, MS 4020, Morgantown, WV 26505, USA. Fax: +1 304285 6126. seo8@cdc.gov (S. Othumpangat). 
mechanisms to respond to virus infection that include the expression of cytokines, MHC class II (Gao et al., 1999) pro-inflammatory molecules, intercellular adhesion molecule-1 (ICAM-1) (Papi and Johnston, 1999a, b), IFN regulatory factor-1 (Uetani et al., 2008), and nitric oxide synthase (Uetani et al., 2000).

ICAM-1 (CD54) belongs to the immunoglobulin superfamily of adhesion molecules, is expressed at low levels in diverse cell types, and is induced by cytokines (TNF-a, interleukin-1) and bacterial lipopolysaccharide (Dustin et al., 1986, 1988). It was originally believed that ICAM-1 played a purely adhesive role, but recent evidence suggests that ICAM-1 can transduce biochemical signals (Matsukura et al., 1996; Matsunaga et al., 1996). In rhinovirus induction of ICAM-1, promoter activity was critically found to be dependent upon up-regulation of NF- $\kappa$ B proteins (Papi and Johnston, 1999b). Though the role of ICAM-1 in rhinovirus has been well studied as the major receptor (Greve et al., 1989; Othumpangat et al., 2012), little has been reported with influenza virus. Here we demonstrate that influenza infection results in up-regulation of ICAM-1 and the nuclear factor- $\kappa \mathrm{B}(\mathrm{NF}-\mathrm{\kappa} \mathrm{B})$ complex which regulates influenza virus replication in airway epithelial cells.

The NF- $\kappa \mathrm{B}$ family of proteins was initially characterized as a group of transcription factors critical to the immune response to pathogens and other foreign bodies. Subsequently, these proteins have been found to regulate expression of a variety of genes responsible for diverse biological processes, including cell proliferation, migration, survival, and stress response. In the basal state, NF- $\kappa \mathrm{B}$ remains in the cytoplasm by interacting with $\mathrm{I} \kappa \mathrm{B}$, an inhibitory protein. I $\kappa \mathrm{B}$ phosphorylation, ubiquitination, and subsequent proteasomal degradation leads to the release of NF- $\mathrm{BB}$ (DiDonato et al., 1997), which then enters the nucleus and activates the expression of pro-inflammatory genes. Thus, diverse upstream signaling pathways converge at the I $\kappa \mathrm{B}$ kinase (IKK) complex, which phosphorylates I $\kappa \mathrm{B}$ that leads to NF- $\kappa \mathrm{B}$ activation. In addition, the expression of the nonstructural 1 (NS1) protein prevent virusand/or double-stranded RNA (dsRNA)-mediated activation of the NF- $\kappa \mathrm{B}$ pathway and depends on the ability of NS1 protein of influenza A virus to bind to dsRNA. NS1-mediated inhibition of the NF- $\kappa$ B pathway may thus play a key role in the pathogenesis of influenza A virus (Wang et al., 2000). Furthermore, it was suggested that NF- $\kappa B$ differentially regulates viral RNA synthesis (Kumar et al., 2008). Here we investigated the role of ICAM-1 modulating the viral copy numbers during the early stages of infection.

Influenza virus induced ICAM-1 gene expression and signaling were investigated in undifferentiated primary human bronchial epithelial cells (HBEpC). Influenza virus infection was found to enhance ICAM-1 expression and induces steady-state messenger RNA (mRNA) levels. The intracellular signaling pathways leading to enhanced ICAM-1 expression appeared to involve the activation of NF- $\kappa \mathrm{B}$. Consequently, NF- $\kappa \mathrm{B}$ activation resulted in the suppression of influenza virus propagation. 


\section{Results}

\section{Influenza virus alters the expression of extracellular matrix and adhesion molecules}

The major site of viral contact with the host and the target of infection and inflammation is the airway epithelium. Airway epithelial cells respond to virus by expressing a variety of inflammatory and immune defense proteins that protect the cells against the virus. In order to understand the immediate response on the cell surface, we infected HBEpC with 3 different strains of influenza virus, H1N1, H3N2 and H9N1, and analyzed extracellular matrix and adhesion proteins using a $\mathrm{RT}^{2}$ profiler PCR array for extracellular matrix and adhesion molecules (Fig. 1). Data showed significant differential expression of several genes including the cell adhesion molecules ICAM-1 (5-7.5 fold) and PECAM-1 (0.6-2 fold) (Fig. 1). Expression of ICAM-1 was highest in cells infected with H3N2 and H9N1 (7.5-8 fold) compared to H1N1 (5.0 fold) strains. The microarray contained a panel of 86 genes for extracellular matrix and adhesion molecules and a significant increase in the expression of metalloproteases (MMPs), protease inhibitors, and integrins were also observed (data not shown). Although there was a significant increase in PECAM-1 in H3N2 and H9N1 strains, it was not comparable to the levels of ICAM-1. ICAM-1 was more consistent across different strains of influenza virus than PECAM-1. Here, we explored the role of ICAM-1 in regulating the survival and replication of influenza virus in host cells was further studied.

\section{Influenza virus induced ICAM-1 expression in primary human bronchial epithelial cells}

The induction of ICAM-1 in HBEpCs exposed to Influenza virus was dependent upon the strains of influenza virus used. Expression of ICAM-1 in influenza infected cells peaked at 3 $\mathrm{h}$ and showed a 7-12 fold increase over mock-infected cells at $6 \mathrm{~h}$ of exposure (Fig. 2A). The PCR array data was reproducible with the highest expression of ICAM1 in H9N1 infected cells after $3 \mathrm{~h}$ of exposure ( 12 fold). Based on the PCR data, the $3 \mathrm{~h}$ time point was chosen for all subsequent studies. Interestingly, the H3N2 strain showed an inverse relationship to the expression of ICAM-1 and the influenza virus copy number (Fig. 2B). A reduction in ICAM-1 expression at $6 \mathrm{~h}$ and an increase in virus matrix copy numbers was observed with $\mathrm{H} 1 \mathrm{~N} 1$ and $\mathrm{H} 3 \mathrm{~N} 2$ strains. The heat inactivated virus failed to induce the expression of ICAM-1 (Fig. 2C) in HBEpC.

The expression of soluble ICAM-1 (sICAM-1) protein released into the culture medium was estimated by ELISA. Data presented in Fig. 3 shows that the expression of sICAM-1 was significantly higher in cells infected with H1N1 $(22 \mathrm{ng} / \mathrm{mL}, p<0.01), \mathrm{H} 3 \mathrm{~N} 2$ infected strains ( $20 \mathrm{ng} / \mathrm{mL}, p<0.05)$, and H9N1 (24.5 ng/mL, $p<0.05)$ compared to the mock infected cells $(15 \mathrm{ng} / \mathrm{mL})$. ICAM-1 surface expression was monitored by flow cytometry and showed an increase in ICAM-1 protein expression compared to the mock infected cells (Fig. 3B). H9N1 infected cells showed the highest increase in ICAM-1 surface expression compared to the other two strains of influenza virus. We further examined the expression of ICAM-1 in cells exposed to different strains of influenza virus and heat inactivated virus to show the active virus with the multiplication only could induce the expression of ICAM-1. Western blot analysis shown in Fig. 3C shows that the ICAM-1 increased on exposure to active virus compared to heat inactivated virus. 
The surface expression of ICAM-1 was further confirmed by confocal microscopy using HBEpCs exposed to H3N2 and H1N1 strains (3 h, MOI=1.0) (Fig. 4). An intense green fluorescence in cells infected with the virus (lower panels) clearly demonstrates the increased expression of ICAM-1 compared to the mock infected cells (upper most panel). The cell integrity in H1N1 (bottom panel) or H3N2 (middle panel) infected cells was examined with phalloidin stain (red) showing disruption in actin filaments in influenza infected cells.

Next we analyzed whether cell type plays a role in influenza induced ICAM-1 expression. Primary human nasal epithelial cells were exposed for $3 \mathrm{~h}$ to influenza A (H1N1, H3N2, or $\mathrm{H} 9 \mathrm{~N} 1)$ at an MOI of 1.0. Nasal epithelial cells upregulated ICAM-1 expression 2.5 fold with H1N1, and 4 fold by H3N2 and H9N1 strains (Fig. 5A). We also evaluated the influenza virus matrix gene copy numbers to confirm the infection efficiency (Fig. 5B). Infection with H3N2 showed the lowest levels of matrix gene copy number as compared to the H1N1 or H9N1 strains and that was comparable to the results obtained with HBEpC. The difference in expression with different cells (nasal vs bronchial) could be due to their physiological difference, although we have not assayed for the nature of sialic acid receptors $(2,3$ or 2, 6) in either cell type.

\section{Silencing ICAM-1 changes the viral copy numbers}

To investigate the role of ICAM-1 in regulating the influenza infection efficiencies in bronchial epithelial cells, epithelial cells were transfected with SCR siRNA or ICAM-1 siRNA and infected with H1N1 strain. As shown in Fig. 6A, ICAM-1 knock-down by siRNA was efficient $(70-80 \%)$ and highly significant $(p<0.001)$ in both uninfected and infected cells compared to the scrambled siRNA transfected cells. Interestingly, the lower expression of ICAM-1 in siRNA ICAM-1 transfected cells showed higher matrix copy numbers (Fig. 6B). There was significantly lower $(p<0.001)$ extracellular secretion of ICAM-1 in cells that were transfected with siRNA ICAM-1 $(4.5 \mathrm{ng} / \mathrm{mL})$ compared to the uninfected siRNA SCR (15.2 ng/mL) (Fig. 6C). Upon infection the sICAM-1 levels were significantly increased in SCR siRNA $(22.0 \mathrm{ng} / \mathrm{mL})$ compared to siRNA ICAM-1 transfected and infected cells $(7.1 \mathrm{ng} / \mathrm{mL})$. Western blot analysis showed a lower expression of ICAM-1 as well as NF- $\kappa$ Bp65 in cells that were treated with siRNA ICAM-1 (Fig. 6D). We further confirmed this in a separate experiment by using a specific ICAM-1 blocking antibody to prevent ICAM-1 from binding to its receptors and to abrogate the ICAM-1 expression. The results showed (Fig. 7) significant up-regulation of the matrix copy numbers in cells treated with the ICAM-1 antibody $(p<0.001)$ compared with control cells treated with the IgG isotype antibody. The inhibitory effect of the antibody on viral replication was less potent than the effect of gene silencing by siRNA studies. Similarly, the inhibitor treatment to reduce ICAM-1 expression was also highly effective compared to the antibody treatment. We used different concentrations $(0-100 \mathrm{ng} / \mathrm{mL})$ of the ICAM-1 inhibitor A 205804 (TOCRIS), whereas $50 \mathrm{ng} / \mathrm{mL}$ showed the highest (100\%) increase in matrix copy number compared to the DMSO treated control cells infected with influenza virus (Fig. 7). 


\section{Effects of IL-1 $\beta$ on influenza virus infection}

In another set of experiments, we added exogenous recombinant human IL- $1 \beta$ to induce ICAM-1 expression (Terajima et al., 1997; Tosi et al., 1992). Different concentrations of exogenous IL- $1 \beta$ (R\&D systems, MN) (50 and $100 \mathrm{ng} / \mathrm{mL})$ were added to confluent layers of HBEpCs and incubated for $2 \mathrm{~h}$. RNA was extracted and the expression of ICAM-1 analyzed. $50 \mathrm{ng} / \mathrm{mL}$ of IL- $1 \beta$ significantly induced ICAM- 1 transcripts by 12 fold while 100 $\mathrm{ng} / \mathrm{mL}$ of IL-1 $\beta$ showed 13 fold increase $(p<0.001)$. Concordant increase in NF- $\kappa$ Bp65 was also observed with both concentrations of IL-1 $1 \beta$. Following treatment with IL-1 $\beta$, cells were exposed to H1N1 strain for another $3 \mathrm{~h}$ at an MOI of 1.0. When normalized to control (treated as 1), the ICAM1 levels (Fig. 8B) were increased 6.2 and 10 fold with IL-1 50 and $100 \mathrm{ng} / \mathrm{mL}$, respectively. Interestingly, the level of NF- $\mathrm{KB}$ was also increased in both treatments and was higher in cells treated with $100 \mathrm{ng} / \mathrm{mL}$ of IL-1 $\beta$. Western blot analysis showed increased expression of ICAM- 1 and NF- $\kappa \mathrm{Bp} 65$ proteins with increasing concentrations of IL-1 $\beta$ (Fig. 8C). Fig. 8D shows IL-1 $\beta(p<0.001)$ treatment caused a significant reduction in influenza A virus matrix gene copy number. To further evaluate the role of ICAM-1, we inhibited the ICAM-1 levels with ICAM-1 chemical inhibitor 205804, and then exposed to 50 and $100 \mathrm{ng} / \mathrm{mL}$ of IL- $1 \beta$. Fig. $8 \mathrm{E}$ shows that the treatment with IL-1 $\beta$ did not alter the copy numbers due to the inhibition of ICAM-1 by the specific inhibitor.

\section{ICAM-1 regulation during influenza virus infection is mediated through NF- $\mathrm{kB}$ activation}

A NF- $\kappa$ B responsive luciferase construct was used (Fig. 9) to examine the ICAM-1 mediated NF- $\kappa$ B response in HBEpC. Cells transfected with siRNA specific for ICAM-1, co-transfected with the NF- $\kappa$ B reporter showed a significant reduction in luciferase activity compared to the SCR siRNA transfected cells (Fig. 9A). The lack of ICAM-1 in cells transfected with ICAM-1 siRNA reduced the activation of NF- $\kappa \mathrm{B}$, On the other hand, infection with influenza virus induced the luciferase activity in SCR siRNA treated cells but showed a partial reduction in luminescence in cells treated with ICAM siRNA (Fig. 9B). These data suggests that a reduction in ICAM-1 levels significantly reduces the NF- $\kappa \mathrm{B}$ activity.

\section{Discussion}

This study provides further evidence that the cellular adhesion molecule ICAM-1 has a significant role in regulating influenza replication and/or survival during the early stages of infection. Our data suggests that up-regulation of ICAM-1 induces expression of NF- $\kappa \mathrm{B}$ protein which further modulates viral replication in the cells in early stages of infection. The primary site of viral contact with the host and the primary target of infection are the airway epithelial cells (Kagnoff and Eckmann, 1997). Among cell lines derived from the human respiratory tract, bronchial and nasal epithelial cells showed significant up-regulation of ICAM-1 upon exposure to influenza virus. Increase in ICAM-1 levels on exposure to influenza virus has been reported in NCI-H292 cells (Matsukura et al., 1996). Airway epithelial cells responded to the viral invaders by expressing a variety of inflammatory and immune defense proteins that defend the cells against the viral infection (Medzhitov and Janeway, 2000). Also, tissue tropism plays a key role in viral infection and induction of inflammatory responses (Meliopoulos et al., 2014). The bronchial cells showed a higher fold 
increase in ICAM-1 expression compared to nasal epithelial cells. Epithelial cells have the influenza binding receptors (2, 3 sialic acid) on their surfaces, but the difference in expression probably is due to the cell physiology as described in a study looking at the infectivity of influenza virus and the differential response to tissues tropism (Zeng et al., 2013). Our data also suggests that different cell types of the respiratory tract respond differently to influenza virus infection.

Influenza virus induced NF- $\kappa \mathrm{B}$ activity, while the reduction in ICAM-1 substantially decreased $N F-\kappa B$ reporter plasmid mediated luciferase activity. If ICAM-1 did not initiate $\mathrm{NF}-\kappa \mathrm{B}$ activation upon viral entry into bronchial cells, then ICAM-1 inhibition would not affect the $N F-\kappa B$ activity and virus replication. Our hypothesis may have important translational implications, as the pharmacological activation of NF- $\kappa \mathrm{B}$, using strategies based on addition of cytokines like IL-1 $\beta$, could favor an induction of ICAM- 1 that alter the phosphorylation and activation of the $\mathrm{NF}-\kappa \mathrm{B}$ pathway, resulting in decreased influenza matrix gene copy number. This notion may offer a promising new approach for the management of influenza virus infection. More importantly, the correlation observed between the patterns of viral replication and ICAM-1 expression of the host cells, together with the evidence that ICAM- 1 is able to regulate the NF- $\kappa \mathrm{B}$ pathway, provides a plausible molecular mechanism to explain both tropism and clinical manifestations of infection.

$\mathrm{NF}-\kappa \mathrm{B}$ comprises a family of transcription factors that play an essential role in the regulation of many physiological responses, ranging from immune and inflammatory responses to cell differentiation and apoptosis (Ghosh et al., 1998). Using reporter gene assays we were able to demonstrate that ICAM-1 has a role in NF- $\kappa$ B pathway in HBEpC. If ICAM-1 modulates extracellular signaling through changes in the expression of ligands and/or receptors, then these changes might affect the $\mathrm{NF}-\kappa \mathrm{B}$ signal cascades. In a study with rhinovirus, ICAM-1 promoter activity was found to involve up-regulation of $\mathrm{NF}-\kappa \mathrm{B}$ proteins (Papi and Johnston, 1999a, b). Similarly, in our studies on exposure to influenza virus the NF- $\kappa$ Bp65 subunits were induced with the increased expression of ICAM-1. The cell surface expression of ICAM-1 protein was also increased in cells infected with the viruses (Fig. 3B). Our data suggests that the mechanism involved in influenza virus mediated induction of ICAM-1 has a role in NF- $\kappa$ B phosphor-ylation which could be one of the mechanism involved in regulating the viral replication in airway epithelial cells. Our findings explain the importance of ICAM-1, a molecule important in inflammatory cell recruitment and activation in asthma (Wegner et al., 1990). We cannot fully dismiss that the other NF-kB dependent mechanisms may be involved in the observed effects, such as expression of the viral NS1 protein counteracting the activation of NF- $\kappa \mathrm{B}$ (Munir et al., 2011) and NF- $\kappa$ B promoter activity (Wang et al., 2000). Heat inactivated virus was failed to induce the expression of ICAM-1, suggesting that active virus are required for the induction of ICAM-1 expression.

Both influenza virus infection and exogenous IL-1 $\beta$ increased ICAM-1 expression on epithelial cells. Addition of IL-1 $\beta$ significantly reduced airway epithelial cells susceptibility to influenza virus infection, possibly through the mechanism of induction of ICAM-1, which in turn, induced the NF- $\kappa$ B pathway. Earlier studies by Terajima et al. (1997) have also shown that the IL-1 $\beta$ increased ICAM-1 expression in primary epithelial cells upon exposure 
to rhinovirus. Interestingly, when ICAM-1 was inhibited by the chemical inhibitor 205804, the cells failed to induce the IL-1 $\beta$ effect, suggesting that ICAM- 1 may have an important role in modulating influenza virus replication.

We also studied the effect of ICAM-1 down-regulation in modulating viral replication in epithelial cells. This was done by blocking ICAM-1 expression with the ICAM-1 specific chemical inhibitor 205804, and the anti-ICAM-1 antibody. As expected, reduced levels of ICAM-1 increased influenza virus infection as measured by the matrix gene copy numbers, suggesting the role of the endogenous ICAM-1 in regulating the influenza virus infection. Up-regulation of ICAM-1 expression on epithelial cells in response to influenza infection may be relevant to the pathogenesis of airway inflammation associated with mechanisms of viral exacerbations of asthma.

In conclusion, our data demonstrate that influenza infection up-regulates ICAM-1 and NF$\kappa \mathrm{B}$ in airway epithelial cells, and represses virus propagation and survival. NF- $\kappa \mathrm{B}$ also exerts apop-totic and inflammatory effects that induce the clearance of infected cells, becoming unfavorable for influenza virus survival. Pharmacological induction of ICAM-1 pathway may have important anti-viral effects and could be beneficial in the management of influenza infection.

\section{Materials and methods}

\section{Cell culture}

HBEpC that are from humans with no underlying diseases were purchased from Promo Cell (Hamberg, Germany), and Cell applications Inc. (San Diego, CA) and sub-cultured as recommended by the supplier in epithelial cell growth media. Human nasal epithelial cells were purchased from Promo cell and maintained by the medium and supplements provided by the supplier. All cells were passaged when $80 \%$ confluent. Cells of lower passage (P 2-6) were used throughout the experiments. Madin-Darby canine kidney (MDCK) cells were used for the propagation of influenza virus. MDCK cells were cultured in EMEM (ATCC) supplemented with $10 \% \mathrm{FBS}$ and $100 \mathrm{IU} / \mathrm{ml}$ penicillin and $100 \mu \mathrm{g} / \mathrm{ml}$ streptomycin sulfate.

\section{Viruses and their infections}

Influenza strain A/Aichi/2/68 (H3N2-ATCC 1680) was purchased from the ATCC and maintained as described (Blachere et al., 2011, 2009). Influenza virus H1N1 (A/WSN/33) was a kind gift from Dr. Robert A. Lamb (Northwestern University, IL), and H9N1 strain was a kind gift from Dr. Daniel Perez (University of Georgia, GA). H3N2 was isolated from human and was deposited with ATCC by M T Coleman, Centers for Disease Control and Prevention (Atlanta, USA). H9N1strain is a genetically modified avian influenza strain of H9N2 by Perez group.

The initial inoculum was added to the cells $\left(\sim 1 \times 10^{6}\right)$ to make a multiplicity of infection (MOI) of 1.0 infection or adjusted appropriately depending on the number of cells in a six well or 96 well plate. All infections of airway epithelial cells were performed in 6-well plates at a dose MOI of 1.0 unless otherwise specified. Control cells were treated in the same manner as infected cells but without the addition of virus (mock). Six well plates were 
seeded with $5 \times 10^{5}$ cells per well and grown to $80 \%$ confluence. Cells were rinsed with PBS, and then virus diluted in modified Hank's Balanced Salt Solution (HBSS) was added to each well. Excess viruses were removed by washing the cells with PBS after 45 min of incubation at $37^{\circ} \mathrm{C}$. Fresh F-12 media was added containing $1 \mu \mathrm{g} / \mathrm{mL}$ of TPCK-trypsin (Sigma-Aldrich, St. Louis, MO) and incubated at $37{ }^{\circ} \mathrm{C}$ and $5 \% \mathrm{CO}^{2}$. TPCK was not added to $\mathrm{A} / \mathrm{WSN} / 33$ virus. Cells were harvested at different time intervals and used for protein and RNA studies.

Influenza virus (H9N1, H3N2, and $\mathrm{H} 9 \mathrm{~N} 1)$ were heat inactivated at $56{ }^{\circ} \mathrm{C}$ for $30 \mathrm{~min}$. The viral plaque assay was performed to confirm the complete inactivation of the virus.

\section{RNA Isolation and QPCR}

Total RNA from airway epithelial cells was isolated using the RNeasy plus Mini kit (Qiagen, MD) according to the manufacturer's instructions. cDNA was generated using the High capacity ABI-RT PCR kit (Life Technologies, CA). Real time qPCR reactions were carried out using the TaqMan FAST PCR reagent (Life Technologies) and the ABI 7500 FAST real-time PCR cycler (Life Technologies). TaqMan primers for ICAM-1, NF- $\kappa$ B and glyceraldehyde phosphate dehydrogenase (GAPDH) were purchased from Life Technologies. Differences in expression were determined using a $\Delta \Delta \mathrm{ct}$ method and GAPDH was used for normalization.

Influenza matrix gene expression was quantified and reported as influenza copy number. QPCR was performed using the TaqMan assay with matrix-specific primers, as reported earlier (Blachere et al., 2011; Spackman et al., 2002). All primers and probes were custom synthesized by Life Technologies. To determine the relative matrix gene copy number from influenza virus strains, a standard curve was generated from the cloned influenza H1N1 matrix gene and analyzed concurrently with RT-PCR reactions (Othumpangat et al., 2014).

\section{PCR microarray}

Total RNA from HBEpC exposed to H1N1, H3N2, or H9N1 was purified using RNeasy kit as described earlier. PCR array for the expression and identification of different extracellular matrix and adhesion molecules that are targeted by the influenza was studied using the human Extracellular matrix and adhesion molecules $\mathrm{RT}^{2}$ Profiler ${ }^{\mathrm{TM}}$ array (Catalog \# PAHS-008z, SABiosciences, Qiagen, MD). Gene expression results were normalized to GAPDH, and data analyzed using the supplier's online software (Qiagen) and the $\Delta \Delta \mathrm{ct}$ method.

\section{ICAM-1 knock-down by siRNA}

The role of ICAM-1 during influenza infection was investigated by silencing ICAM-1 gene expression in epithelial cells with ICAM-1 specific siRNA. HBEpC were transfected with ICAM1 siRNA or a scrambled oligonucleotide (SCR) (Dharmacon, Thermoscientific, CA) using the lipid-based Lipofectamine 2000 reagent diluted in Opti-MEM-I reduced serum medium (Life Technologies) according to the supplier's protocol. Briefly, HBEpC were grown to $80 \%$ confluence in 6-well plates and the transfection complexes were made in Opti-MEM and directly applied to the cells to a final concentration of 25 or $50 \mathrm{nM}$. After 6- 
$8 \mathrm{~h}$ of transfection, the medium was replaced with fresh complete medium. After 24-36 h transfected cells were exposed to influenza virus (1.0 MOI), according to standard infection protocols. Following virus challenge, cells were collected by trypsinization and the resulting pellet was used for RNA extraction or protein for Western blot analysis.

\section{ICAM-1 quantification by ELISA}

The changes in secreted ICAM-1 protein during influenza virus infection were quantitated using a commercially available ELISA kit (R\&D systems, MN). HBEpC were grown to confluence in 6-well plates and infected with MOI of 1.0 H1N1 (WSN), H3N2, and H9N1 for $3 \mathrm{~h}$. Supernatants were collected following infection and centrifuged to remove cell debris. ELISA for ICAM-1 was completed according to the manufacturer's instructions. The plates were read using a multiwall plate reader (Bio-Tek Instruments Inc., VT) and analyzed using the software of Gen5-2.0 (Bio-Tek).

\section{Flow cytometry}

HBEpC were grown to confluence in 6-well plates and infected with MOI of 1.0 of H1N1 (WSN), H3N2, and H9N1 for $3 \mathrm{~h}$. Cells were isolated by trypsinization and were stained for surface detection of ICAM-1 protein using ICAM-1 specific antibody. ICAM-1 (CD54) mouse monoclonal antibody and the matching isotype control were purchased from (BD Biosciences, San Jose, CA). Secondary fluorescent antibody labeled with Alexa 555 was from Life Technologies (Carlsbad, CA). Data were acquired and analyzed using FACS LSRII (BD Biosciences).

\section{Imaging with confocal microscopy}

HBEpCs were grown on chamber slides overnight (Chamber slide ${ }^{\mathrm{TM}}$, Lab-TekII, Thermo Fisher Scientific, Rochester, NY) or until 80-90\% confluence. Cells were then infected for 3 h with H1N1 or H3N2 strains or an uninfected control. Subsequently, cells were washed with PBS, and fixed with 4\% methanol-free formaldehyde (Polysciences Inc., Warrington, PA). Immunofluorescent staining was done as described earlier (Othumpangat et al., 2013) and stained with rabbit anti-ICAM1 antibody (Millipore, Billerica, MA) for $1 \mathrm{~h}$, followed by Alexa-488 conjugated anti-rabbit secondary antibody and Phalloidin (Life Technologies). The slides were mounted with DAPI-Prolong Gold anti-fade reagent (Life Technologies) and protected with cover slips. Photomicrographs were made using a Zeiss LSM510 (Carl Zeiss, Obertochen, AG Germany) confocal microscope.

\section{Western immunoblotting}

HBEpCs were mock infected or infected at an MOI of 1.0 with H1N1 (WSN), H3N2 and H9N1 or the heat inactivated virus. Three hours postinfection (p.i.), cells were lysed in 100 $\mu \mathrm{l}$ of radio-immunoprecipitation assay (RIPA) buffer (Thermo Scientific, MA) containing the protease inhibitor cocktail. Thirty micrograms of protein was solubilized in protein sample buffer and subjected to electrophoresis on $10 \%$ or 4-20\% precast gradient SDSpolyacrylamide gels (SDS-PAGE, Bio-Rad, CA). Separated proteins were transferred to a nitrocellulose membrane (Millipore, MA). Membranes were blocked using Odyssey Blocking Buffer (LI-COR Biosciences, Lincoln, NE) for $1 \mathrm{~h}$ at room temperature and 
subjected to Western analysis with rabbit polyclonal anti-ICAM-1 (CD54, BD Biosciences), mouse monoclonal anti NF- $k$ Bp65 (EMD Millipore, MA) and mouse monoclonal antiGAPDH (Abcam, MA). Appropriate mouse and rabbit IR Dye 680 or 800 secondary antibodies (LI-COR Biosciences) were used. Near-infrared fluorescence detection was performed on the Odyssey Imaging System (LI-COR Biosciences, Lincoln, NE).

To understand whether the up-regulation of ICAM-1 mRNA expression alters influenza infection efficiency, exogenous IL- $1 \beta$ was added (50 or $100 \mathrm{ng} / \mathrm{ml}$; R\&D systems) to HBEpCs. The cells were incubated for $2 \mathrm{~h}$ with IL-1 $\beta$ prior to virus challenge. Protein was isolated from the treated cells and subjected to Western blot analysis.

\section{NF- $\times B$ reporter assay}

NF- $\kappa B$ cignal reporter kit (Cignal ${ }^{\mathrm{TM}}$ Reporter Assay, SABiosciences, Qiagen, MD) was used to assess NF- $\kappa$ B activity in HBEpC. The kit contains the NF- $\kappa B$ responsive luciferase construct which encodes the firefly luciferase reporter gene under the control of a minimal CMV promoter and tandem repeats of the NF- $\kappa \mathrm{B}$ transcriptional response element (TRE). Briefly, cells $\left(1 \times 10^{4}\right.$ cells/well $)$ were plated on a 96-well plate containing the transfection agent (Lipofectamine 2000, Life Technologies), the NF- $\kappa$ B reporter, and co-transfected with the siRNA for ICAM1 and the scrambled siRNA $(50 \mathrm{nM})$. After $16 \mathrm{~h}$ of transfection, the transfection media was replaced with regular growth media and incubated for 16-18 h. Subsequently, the cells were exposed to influenza virus H1N1 for $3 \mathrm{~h}$. NF- $\kappa B$ activity was assessed using the luciferase assay system (Dual-Glo ${ }^{\circledR}$ Luciferase Assay, Promega, WI). Cells were lysed with the passive lysis buffer (provided in the kit) before luminescence was detected with a Glomax ${ }^{\circledR} 96$ well microplate Luminometer (Promega). NF- $\kappa$ B activity was standardized to the transfection of each cell by normalizing data to Renilla luminescence.

\section{Statistical analysis}

Analysis of variance (ANOVA) was used to analyze qPCR, and reporter assay data. The post-hoc pairwise comparisons between means were performed using the Holm-Sidak method with a $p$ value of $<0.05$ considered statistically significant (Sigmaplot version 12.5 for Windows, Systat Software, Chicago, IL).

\section{Acknowledgments}

The findings and the conclusions in this report are those of the authors and do not necessarily represent the views of the National Institute for Occupational Safety and Health.

\section{References}

Blachere FM, Cao G, Lindsley WG, Noti JD, Beezhold DH. Enhanced detection of infectious airborne influenza virus. J Virol Methods. 2011; 176:120-124. [PubMed: 21663766]

Blachere FM, Lindsley WG, Pearce TA, Anderson SE, Fisher M, Khakoo R, Meade BJ, Lander O, Davis S, Thewlis RE, Celik I, Chen BT, Beezhold DH. Measurement of airborne influenza virus in a hospital emergency department. Clin Infect Dis. 2009; 48:438-440. [PubMed: 19133798]

DiDonato JA, Hayakawa M, Rothwarf DM, Zandi E, Karin M. A cytokine-responsive IkappaB kinase that activates the transcription factor NF-kappaB. Nature. 1997; 388:548-554. [PubMed: 9252186]

Doherty PC, Turner SJ, Webby RG, Thomas PG. Influenza and the challenge for immunology. Nat Immunol. 2006; 7:449-455. [PubMed: 16622432] 
Dustin ML, Rothlein R, Bhan AK, Dinarello CA, Springer TA. Induction by IL 1 and interferongamma: tissue distribution, biochemistry, and function of a natural adherence molecule (ICAM-1). J Immunol. 1986; 137:245-254. [PubMed: 3086451]

Dustin ML, Singer KH, Tuck DT, Springer TA. Adhesion of T lymphoblasts to epidermal keratinocytes is regulated by interferon gamma and is mediated by intercellular adhesion molecule 1 (ICAM-1). J Exp Med. 1988; 167:1323-1340. [PubMed: 3128630]

Gao J, De BP, Banerjee AK. Human parainfluenza virus type 3 up-regulates major histocompatibility complex class I and II expression on respiratory epithelial cells: involvement of a STAT1- and CIITA-independent pathway. J Virol. 1999; 73:1411-1418. [PubMed: 9882346]

Ghosh S, May MJ, Kopp EB. NF-kappa B and Rel proteins: evolutionarily conserved mediators of immune responses. Annu Rev Immunol. 1998; 16:225-260. [PubMed: 9597130]

Greve JM, Davis G, Meyer AM, Forte CP, Yost SC, Marlor CW, Kamarck ME, McClelland A. The major human rhinovirus receptor is ICAM-1. Cell. 1989; 56:839-847. [PubMed: 2538243]

Kagnoff MF, Eckmann L. Epithelial cells as sensors for microbial infection. J Clin Investig. 1997; 100:6-10. [PubMed: 9202050]

Kumar N, Xin ZT, Liang Y, Ly H. NF-kappaB signaling differentially regulates influenza virus RNA synthesis. J Virol. 2008; 82:9880-9889. [PubMed: 18701591]

Matsukura S, Kokubu H, Noda H, Watanabe K, Fukuchi K, Gomi M, Adachi. Expression of ICAM-1 on human bronchial epithelial cells after influenza virus infection. Allergol Int. 1996; 45:97-103.

Matsunaga T, Katayama I, Yokozeki H, Nishioka K. ICAM-1 expression on keratinocytes in mechanically-injured skin of a patient with atopic dermatitis. J Dermatol Sci. 1996; 12:219-226. [PubMed: 8884526]

Medzhitov R, Janeway C Jr. Innate immunity. N Engl J Med. 2000; 343:338-344. [PubMed: 10922424]

Meliopoulos VA, Karlsson EA, Kercher L, Cline T, Freiden P, Duan S, Vogel P, Webby RJ, Guan Y, Peiris M, Thomas PG, Schultz-Cherry S. Human H7N9 and H5N1 influenza viruses differ in induction of cytokines and tissue tropism. J Virol. 2014; 88:12982-12991. [PubMed: 25210188]

Munir M, Zohari S, Berg M. Non-structural protein 1 of avian influenza A viruses differentially inhibit NF-kappaB promoter activation. Virol J. 2011; 8:383. [PubMed: 21810221]

Othumpangat S, Noti JD, Beezhold DH. Lung epithelial cells resist influenza A infection by inducing the expression of cytochrome c oxidase VIc which is modulated by miRNA 4276. Virology. 2014; 468-470:256-264.

Othumpangat S, Noti JD, Blachere FM, Beezhold DH. Expression of non-structural-1A binding protein in lung epithelial cells is modulated by miRNA-548an on exposure to influenza A virus. Virology. 2013; 447:84-94. [PubMed: 24210102]

Othumpangat S, Regier M, Piedimonte G. Nerve growth factor modulates human rhinovirus infection in airway epithelial cells by controlling ICAM-1 expression. Am J Physiol Lung Cell Mol Physiol. 2012; 302:L1057-L1066. [PubMed: 22427528]

Papi A, Johnston SL. Respiratory epithelial cell expression of vascular cell adhesion molecule-1 and its up-regulation by rhinovirus infection via NF-kappaB and GATA transcription factors. J Biol Chem. 1999a; 274:30041-30051. [PubMed: 10514490]

Papi A, Johnston SL. Rhinovirus infection induces expression of its own receptor intercellular adhesion molecule 1 (ICAM-1) via increased NF-kappaB-mediated transcription. J Biol Chem. 1999b; 274:9707-9720. [PubMed: 10092659]

Spackman E, Senne DA, Myers TJ, Bulaga LL, Garber LP, Perdue ML, Lohman K, Daum LT, Suarez DL. Development of a real-time reverse transcriptase PCR assay for type A influenza virus and the avian H5 and H7 hemagglutinin subtypes. J Clin Microbiol. 2002; 40:3256-3260. [PubMed: 12202562]

Stohr K. Influenza - WHO cares. Lancet Infect Dis. 2002; 2:517. [PubMed: 12206966]

Terajima M, Yamaya M, Sekizawa K, Okinaga S, Suzuki T, Yamada N, Nakayama K, Ohrui T, Oshima T, Numazaki Y, Sasaki H. Rhinovirus infection of primary cultures of human tracheal epithelium: role of ICAM-1 and IL-1beta. Am J Physiol. 1997; 273:L749-L759. [PubMed: 9357849] 
Tosi MF, Stark JM, Smith CW, Hamedani A, Gruenert DC, Infeld MD. Induction of ICAM-1 expression on human airway epithelial cells by inflammatory cytokines: effects on neutrophilepithelial cell adhesion. Am J Respir Cell Mol Biol. 1992; 7:214-221. [PubMed: 1353976]

Uetani K, Der SD, Zamanian-Daryoush M, de La Motte C, Lieberman BY, Williams BR, Erzurum SC. Central role of double-stranded RNA-activated protein kinase in microbial induction of nitric oxide synthase. J Immunol. 2000; 165:988-996. [PubMed: 10878375]

Uetani K, Hiroi M, Meguro T, Ogawa H, Kamisako T, Ohmori Y, Erzurum SC. Influenza A virus abrogates IFN-gamma response in respiratory epithelial cells by disruption of the Jak/Stat pathway. Eur J Immunol. 2008; 38:1559-1573. [PubMed: 18493979]

Wang X, Li M, Zheng H, Muster T, Palese P, Beg AA, Garcia-Sastre A. Influenza A virus NS1 protein prevents activation of NF-kappaB and induction of alpha/beta interferon. J Virol. 2000; 74:1156611573. [PubMed: 11090154]

Wegner CD, Gundel RH, Reilly P, Haynes N, Letts LG, Rothlein R. Intercellular adhesion molecule-1 (ICAM-1) in the pathogenesis of asthma. Science. 1990; 247:456-459. [PubMed: 1967851]

Zeng H, Goldsmith CS, Maines TR, Belser JA, Gustin KM, Pekosz A, Zaki SR, Katz JM, Tumpey TM. Tropism and infectivity of influenza virus, including highly pathogenic avian H5N1 virus, in ferret tracheal differentiated primary epithelial cell cultures. J Virol. 2013; 87:2597-2607. [PubMed: 23255802] 


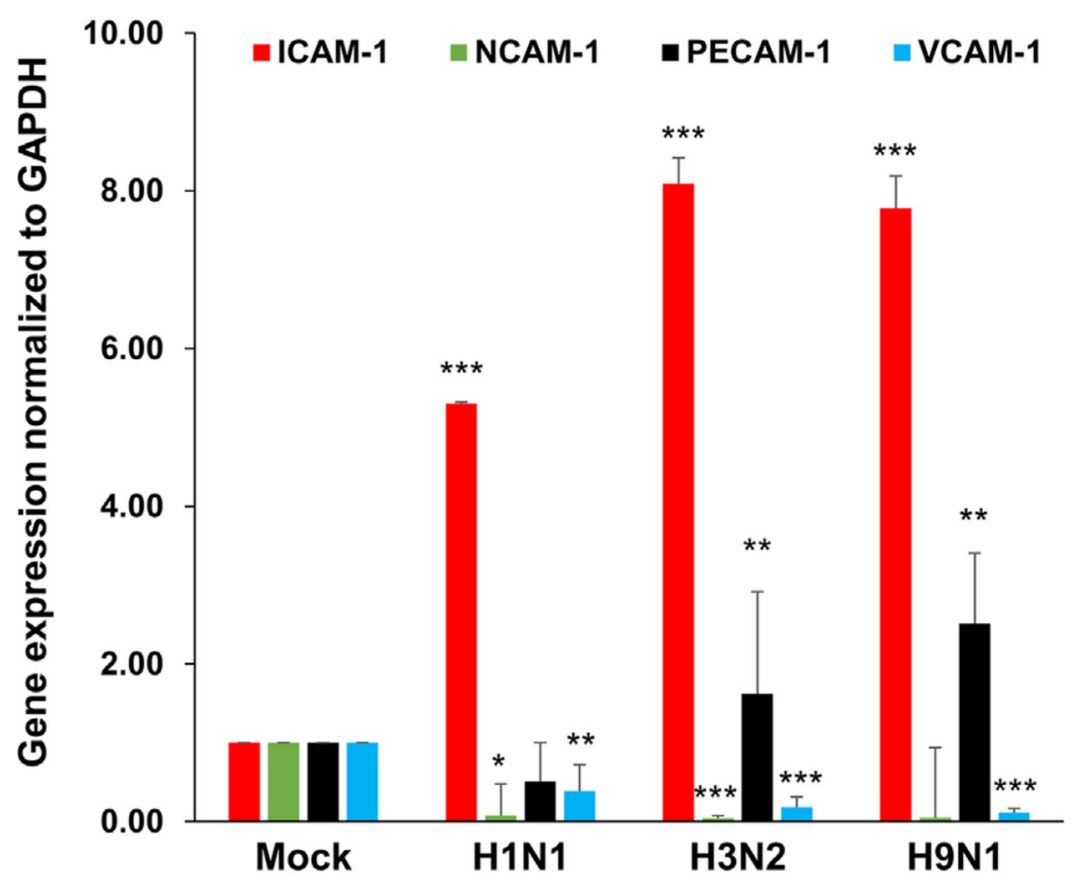

Fig. 1.

Influenza virus alters the cell adhesion and extracellular molecules. PCR array analyses of HBEPC exposed to influenza virus and the expression of extra-cellular matrix cell adhesion molecules ICAM-1, NCAM-1 (Neural cell adhesion molecule-1), PECAM-1 (Platelet/ endothelial cell adhesion molecule-1), and VECAM-1 (Vascular cell adhesion molecule-1). HBEpC were infected with influenza viruses, H1N1, H3N2, and H9N1 with an MOI of 1.0 for $3 \mathrm{~h}$. Total RNA was isolated and subjected to the human extracellular matrix and adhesion molecules $\mathrm{RT}^{2}$ Profiler ${ }^{\mathrm{TM}} \mathrm{PCR}$ array (Qiagen). The data are representative of 3 independent experiments $(N=3)$. Data are presented as the means $\pm \mathrm{SE}, * * * p<0.001$, $* * p<0.01$ compared with mock controls. 

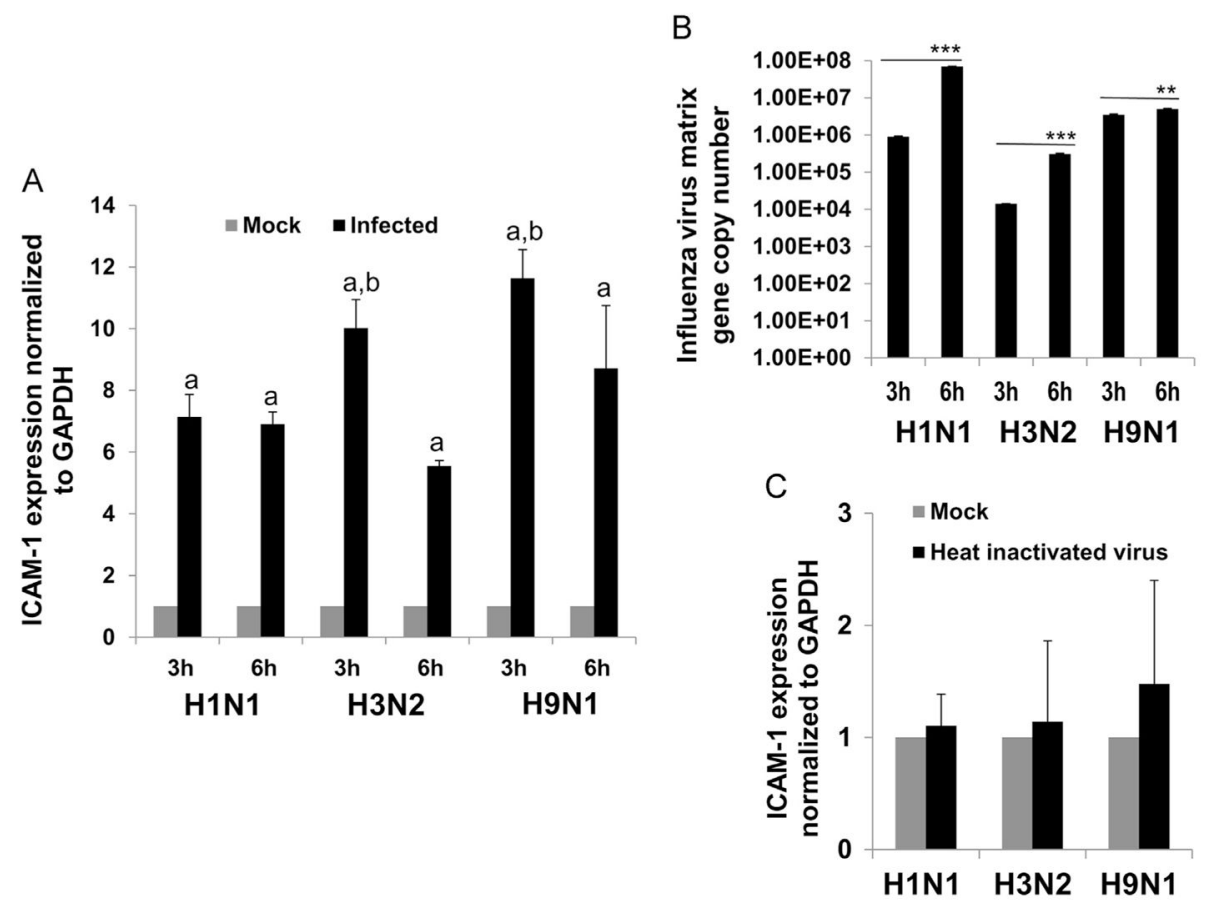

Fig. 2.

Influenza virus induced up-regulation of ICAM-1 mRNA. Confluent HBEpC monolayers were infected with different strains of influenza virus $(\mathrm{H} 1 \mathrm{~N} 1, \mathrm{H} 3 \mathrm{~N} 2$, and H9N1) for $6 \mathrm{~h}$ with an MOI of 1.0, and samples were analyzed at $3 \mathrm{~h}$ and $6 \mathrm{~h}$ postinfection. (A) ICAM-1 expression in response to H1N1, H3N2 and H9N1. Expression of ICAM-1 was analyzed by RT-PCR. (B) Influenza virus matrix gene copy number was analyzed from the RNA extracted from HBEpC infected with influenza virus. (C) ICAM-1 expression in response to heat inactivated $\mathrm{H} 1 \mathrm{~N} 1, \mathrm{H} 3 \mathrm{~N} 2$ and $\mathrm{H} 9 \mathrm{~N} 1$. Virus were heat inactivated at $56{ }^{\circ} \mathrm{C}$ for $30 \mathrm{~min}$ and HBEPC exposed to the virus for $3 \mathrm{~h}$. The expression of ICAM-1 was analyzed by RTPCR. The data are representative of 3 to 4 independent experiments. Data shown as mean $\pm \mathrm{SE}$ ( $n=4$ experiments for RNA isolation and $n=3$ experiments for plaque assay). The different letters (a or b) represents the significant difference within groups $(3 \mathrm{~h}$ and $6 \mathrm{~h}$ ), and the letter ' $a$ ' shows the significant difference compared to the control. $* * * p<0.001$, $* * p<0.01$ compared with mock. 
A Secreted ICAM-1

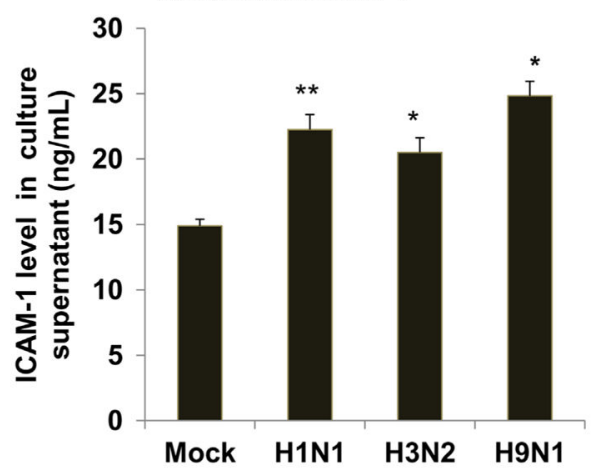

C

Western blot

Heat inactivated Active virus

H1N1 H3N2 H9N1 $\overline{H 1 N 1 \text { H3N2 H9N1 }}$

ICAM-1
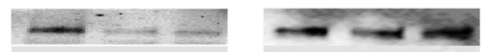

GAPDH

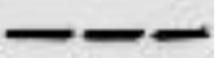

Fig. 3.

Influenza virus induced secretion of soluble ICAM-1 protein. (A) HBEpC were exposed to MOI of 1.0 H1N1, H3N2, or H9N1 influenza virus or control cells mock infected for $3 \mathrm{~h}$. Culture supernatant was collected and subjected to ELISA specific for sICAM-1 and quantified using a standard curve. (B) ICAM-1 cell surface protein expression was measured by flow cytometry after staining HBEpCs infected with MOI of $1.0 \mathrm{H} 1 \mathrm{~N} 1, \mathrm{H} 3 \mathrm{~N} 2$, and H9N1 for $3 \mathrm{~h}$, and uninfected control cells, with ICAM-1 primary antibody and Alexa 555 secondary antibody. (C) Western blot showing the increased expression of ICAM-1 protein in cells infected with active influenza virus compared to the heat inactivated virus strains. Data shown as mean \pm SEM for experiments performed in triplicate $(* * p<0.01, * p<0.05)$ compared to the mock. $(N=3)$. 


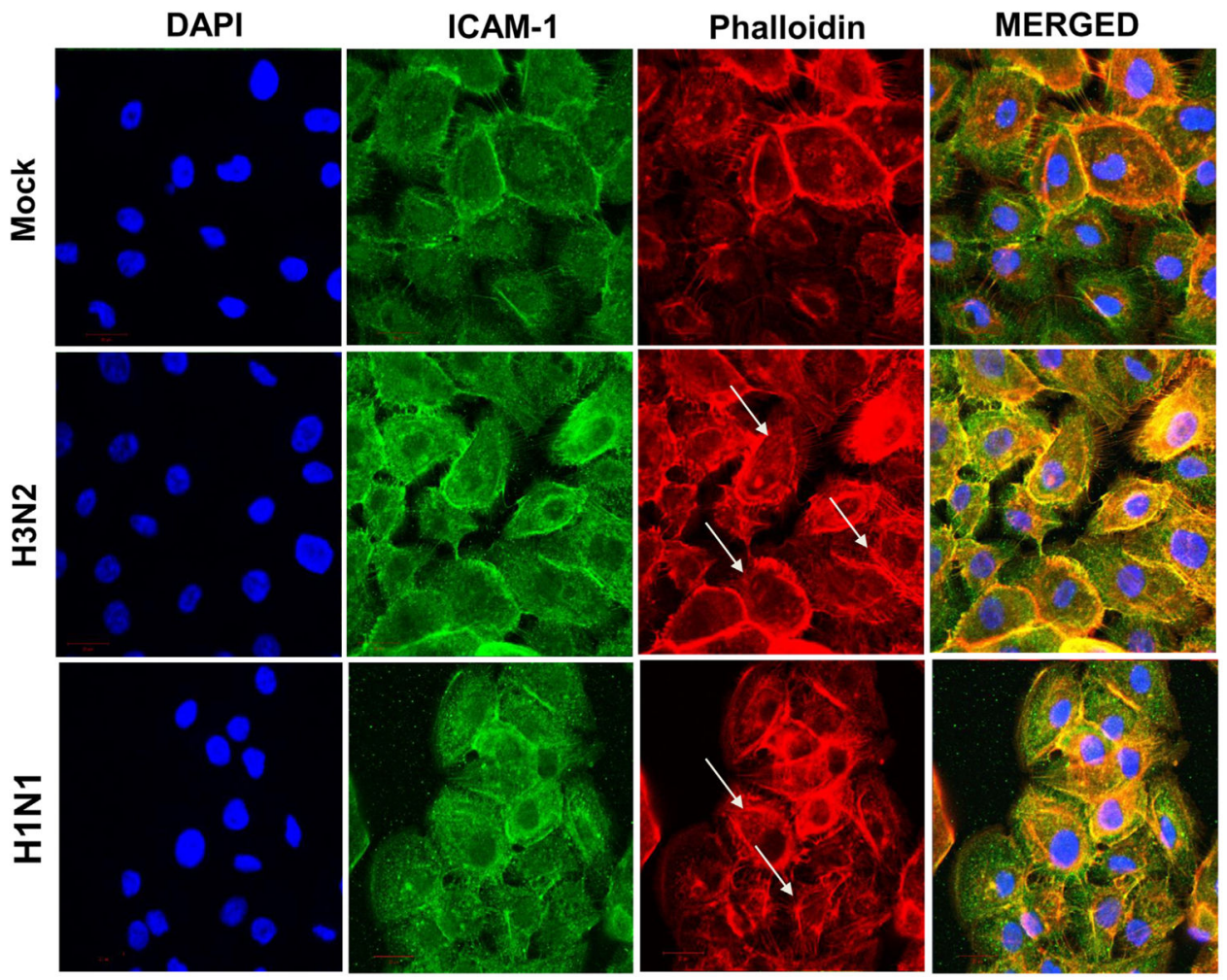

Fig. 4.

Induction of ICAM-1 expression by influenza virus in $\mathrm{HBEpC}$ as detected by immunofluorescence. HBEpC were infected with $\mathrm{H} 3 \mathrm{~N} 2$ and $\mathrm{H} 1 \mathrm{~N} 1$ at an MOI of 1.0 or mock infected. After virus adsorption for $45 \mathrm{~min}$ at $37^{\circ} \mathrm{C}$, the cells were incubated for $3 \mathrm{~h}$. Thereafter, cells were washed, fixed, and processed for immunofluorescence staining of ICAM-1. Lane 1 shows the uninfected control cells. Lane 2 showing the Influenza H3N2 and lane 3 showing H1N1 infected cells. White arrows indicate the difference in morphology of the cells exposed to virus. Cells were stained with anti-ICAM-1 antibodies (green), actin filaments stained by phalloidin (red), and the nucleus by DAPI (blue) observed under the LSM-510 confocal microscope (63X-objective). 

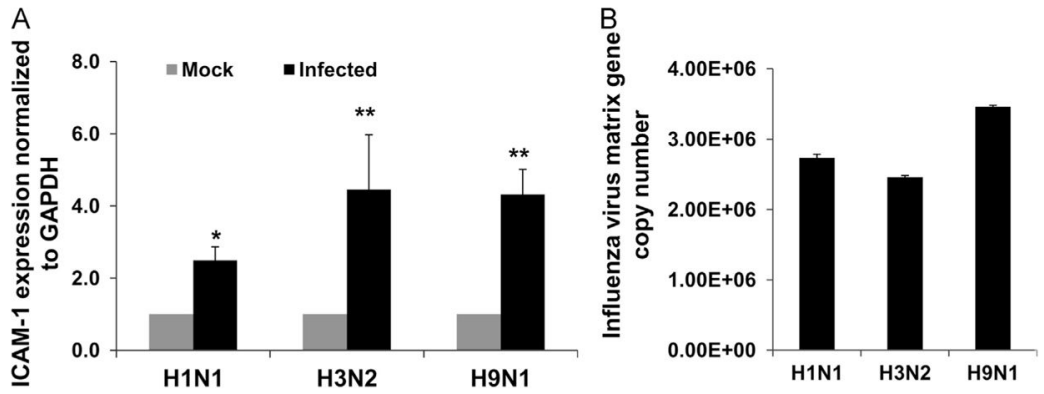

Fig. 5.

ICAM-1 expression in human primary nasal cells. Nasal epithelial cells were infected with influenza virus H1N1 (WSN), H3N2, and H9N1 for $3 \mathrm{~h}$. (A) The expression of ICAM-1 mRNA in nasal epithelial cells infected with H1N1 (WSN), H3N2, and H9N1 at an MOI of 1.0. Fold change was normalized with housekeeping gene GAPDH. (B) Influenza virus matrix gene copy number was analyzed from the RNA extracted from nasal cells infected with $\mathrm{H} 1 \mathrm{~N} 1, \mathrm{H} 3 \mathrm{~N} 2$, and $\mathrm{H} 9 \mathrm{~N} 1$ strains of influenza virus. The data are representative of 3 independent experiments $(N=3)$. Data are presented as the means $\pm \mathrm{SE}, * * p<0.01,{ }^{*} p<0.05$ compared with independent mock controls. 

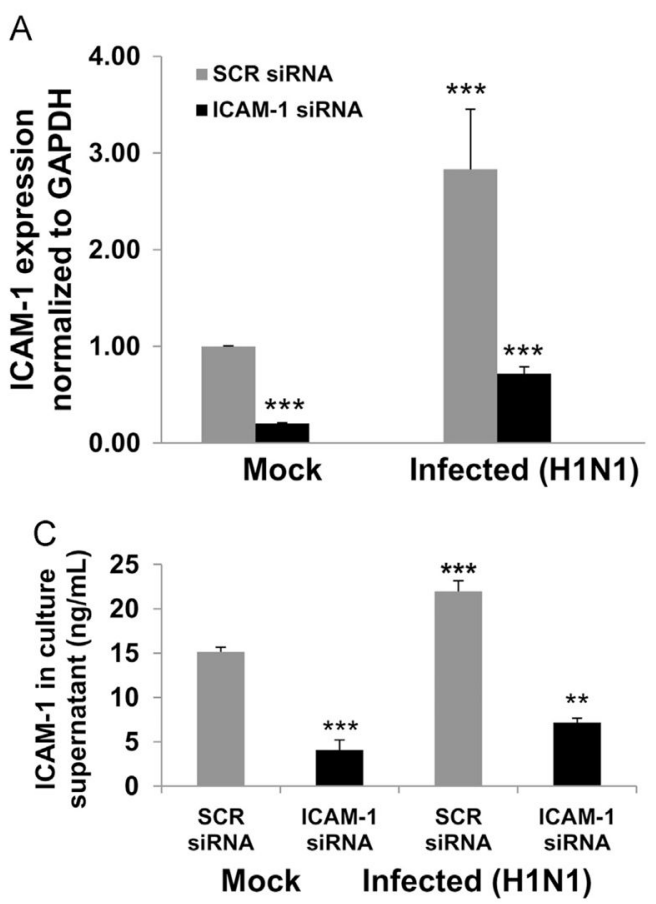

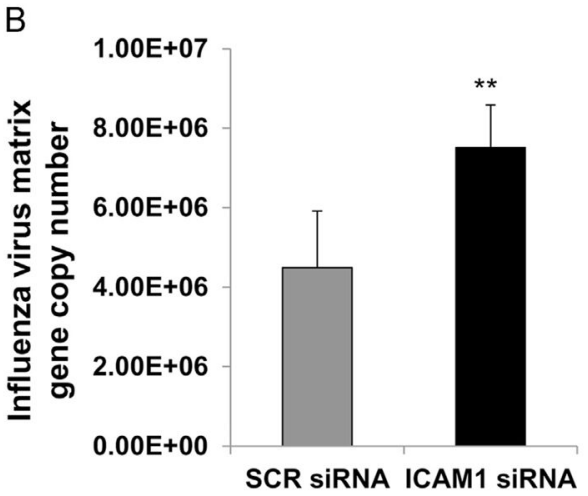

D

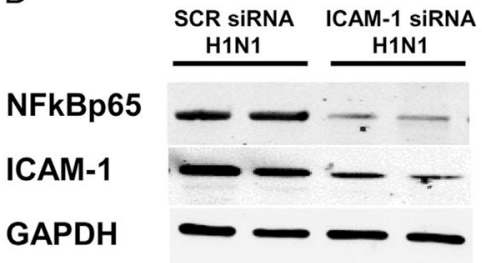

Fig. 6.

Increased viral copy numbers in bronchial cells lacking ICAM-1. HBEpC transfected with ICAM-1 specific siRNA or a scrambled siRNA control for 32-36 h and subsequently exposed to H1N1 (WSN) for another 3 h. (A) ICAM-1 mRNA expression in cells transfected with siRNA ICAM-1 or SCR siRNA and infected with influenza virus or left uninfected. (B) Matrix copy number of influenza virus in cells infected after transfected with siRNA ICAM-1 or SCR siRNA. (C) sICAM-1 levels in cell supernatants collected from cells transfected with SCR siRNA or ICAM-1 siRNA and infected with MOI of 1.0 influenza virus. (D) Western blot showing the reduction in ICAM-1 and NF-kBp65 in cells transfected with ICAM-1 siRNA and infected with MOI of 1.0 influenza virus. The data are representative of 3 independent experiments $(N=3)$. Data are presented as the means $\pm \mathrm{SE}$, $* * * p<0.001, * * p<0.01$ compared with SCR siRNA controls. 


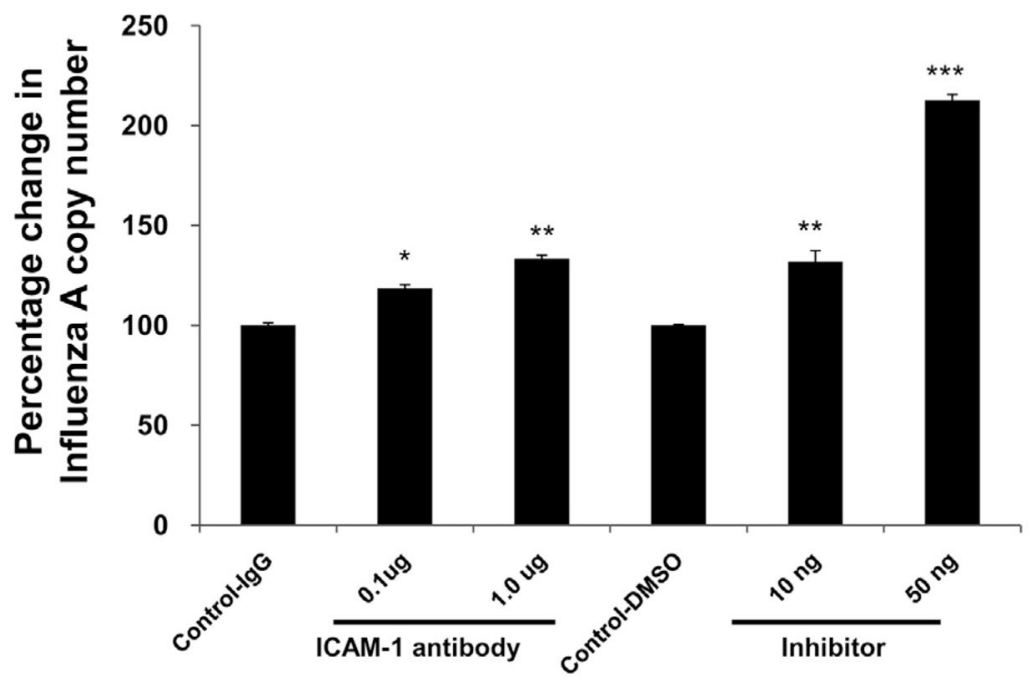

Fig. 7.

ICAM-1 inhibitors induced influenza matrix gene copy number. HBEpC were treated with anti-ICAM-1 antibody ( 0.1 or $1.0 \mu \mathrm{g} / \mathrm{mL})$ or with a matched isotype antibody control for $2 \mathrm{~h}$ and then infected with influenza A H1N1 for an additional $3 \mathrm{~h}$. HBEpC were also treated with ICAM-1 inhibitor-A 205804 (10 and $50 \mathrm{ng} / \mathrm{mL}$ ) (TOCRIS) or a DMSO control for $2 \mathrm{~h}$ and then infected with influenza A H1N1 for additional $3 \mathrm{~h}$. Matrix gene copy number was measured by RT-PCR from the RNA extracted from cells infected with influenza virus or mock. The data are representative of 3 independent experiments $(N=3)$. Data are presented as the means $\pm \mathrm{SE}, * * * p<0.001, * * p<0.01, * p<0.05$ compared with IgG controls or DMSOcontrols. 
A

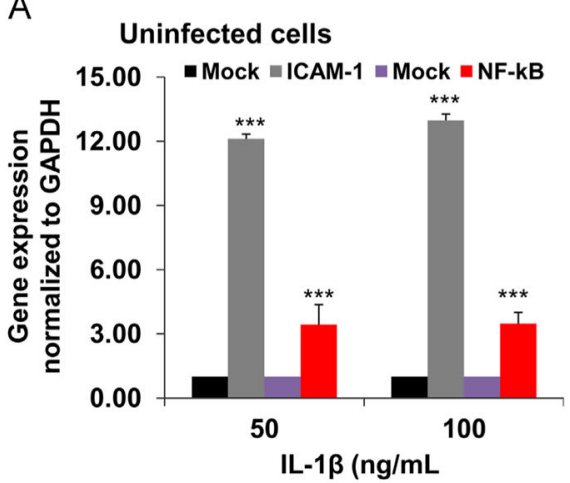

C

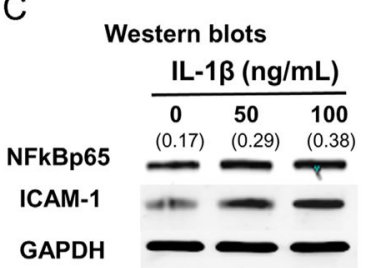

D
B

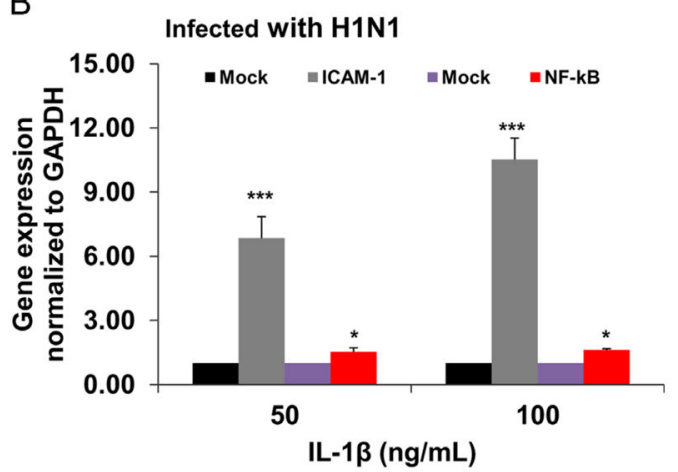
Copy number

E

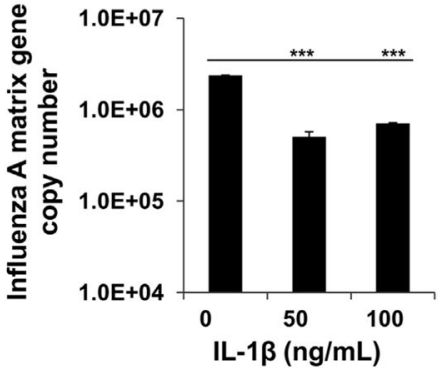

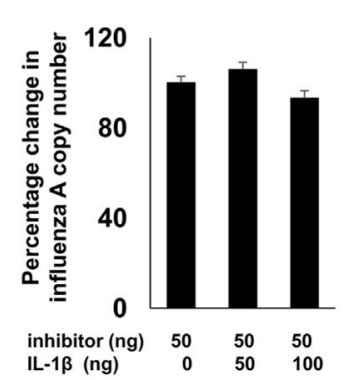

Fig. 8.

Recombinant IL-1 $\beta$ induced ICAM-1 expression in bronchial epithelial cells. (A) IL-1 $\beta$ (50 and $100 \mathrm{ng} / \mathrm{mL}$ ) was added to confluent layers of HBEpC and after a period of $2 \mathrm{~h}$, RNA extracted and analyzed the expression of ICAM-1 and NF- $\kappa$ B. (B) HBEpC were treated with IL-1 $\beta$ for a period of $2 \mathrm{~h}$, and then infected with H1N1 of an MOI of 1.0 for $3 \mathrm{~h}$. RNA extracted and analyzed the expression of ICAM-1 and NF- $\kappa$ B. (C) Western blot analysis showing the changes in NF-kBp65 and ICAM-1 levels in IL-1 $\beta$ treated and untreated HBEpC infected with influenza H1N1 for $3 \mathrm{~h}$. The numbers in parenthesis shows the expression levels NF- $\kappa$ Bp65 quantified and normalized to GAPDH. (D) Matrix copy number of influenza virus in cells treated with IL-1 $\beta$ and then infected with H1N1 for $3 \mathrm{~h}$. (E) Blocking ICAM-1 prevented IL-1 $\beta$ mediated reduction in copy number. HBEpC were treated with ICAM-1 inhibitor-A 205804 (50 ng/mL) (TOCRIS) for $2 \mathrm{~h}$. Subsequently the cells were treated with IL-1 $\beta$ ( 50 or $100 \mathrm{ng} / \mathrm{mL}$ ) for another $2 \mathrm{~h}$ followed by influenza A $\mathrm{H} 1 \mathrm{~N} 1$ for $3 \mathrm{~h}$. The data are representative of 3 independent experiments. Data are presented as the means $\pm \mathrm{SE}, * * * p<0.001, * * p<0.01$ compared with mock or infected controls without IL-1 $\beta$. 


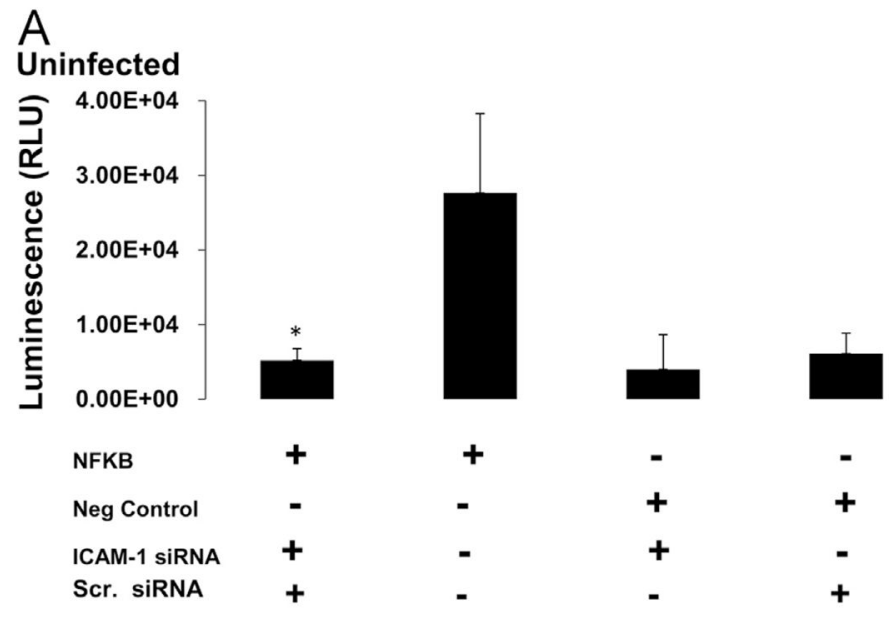

\section{Bnfected with H1N1}

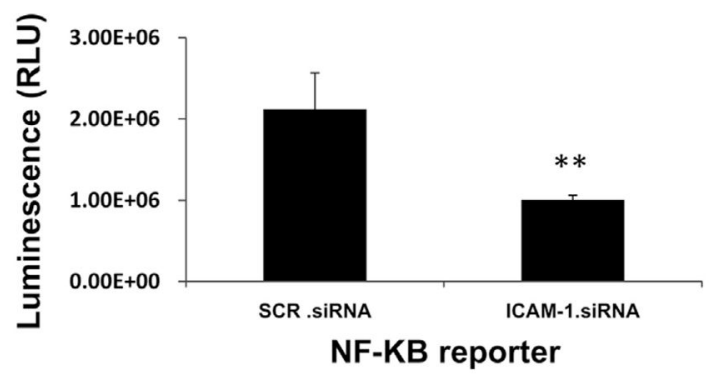

Fig. 9.

Lack of ICAM-1 Inhibit NF- $\kappa \mathrm{B}$ activation on exposure to influenza virus. HBEpC were transiently transfected with $\mathrm{NF}-\kappa \mathrm{B}$ expression vector (100 ng), the $\mathrm{NF}-\kappa \mathrm{B}$ reporter plasmid pELAM-luc $(0.5 \mu \mathrm{g})$, or the negative control expression plas-mid, along with ICAM-1 siRNA or a scrambled siRNA. After $36 \mathrm{~h}$, luciferase levels were determined. The values indicated represent normalized luciferase activities relative to the control transfection using the reporter plasmid and an empty expression vector. (A) HBEpC were transfected with reporter plasmid and siRNA. (B) After transfection with the NF-kB reporter plasmids and siRNA ICAM-1 and SCR siRNA were infected with H1N1 MOI of 1.0 for $3 \mathrm{~h}$. Renilla luciferase activity was measured using a Promega Glomax luminometer, and the data were normalized with firefly luciferase activity. Data shown as mean \pm SEM for experiments performed in triplicate. The data are representative of 3 independent experiments $(N=3)$. Data are presented as the means $\pm \mathrm{SE}, * * p<0.01, * p<0.05$ compared with scrambled siRNA controls. 